\title{
Decoupling Braided Tensor Factors *
}

\author{
Gaetano Fiore, ${ }^{1,2}$ Harold Steinacker ${ }^{3}$, Julius Wess ${ }^{3,4}$ \\ ${ }^{1}$ Dip. di Matematica e Applicazioni, Fac. di Ingegneria \\ Università di Napoli, V. Claudio 21, 80125 Napoli \\ ${ }^{2}$ I.N.F.N., Sezione di Napoli, \\ Complesso MSA, V. Cintia, 80126 Napoli \\ ${ }^{3}$ Sektion Physik, Ludwig-Maximilian Universität, \\ Theresienstraße 37, D-80333 München \\ ${ }^{4}$ Max-Planck-Institut für Physik \\ Föhringer Ring 6, D-80805 München
}

\begin{abstract}
We briefly report on our result [9] that the braided tensor product algebra of two module algebras $\mathcal{A}_{1}, \mathcal{A}_{2}$ of a quasitriangular Hopf algebra $H$ is equal to the ordinary tensor product algebra of $\mathcal{A}_{1}$ with a subalgebra isomorphic to $\mathcal{A}_{2}$ and commuting with $\mathcal{A}_{1}$, provided there exists a realization of $H$ within $\mathcal{A}_{1}$. As applications of the theorem we consider the braided tensor product algebras of two or more quantum group covariant quantum spaces or deformed Heisenberg algebras.
\end{abstract}

*Talk given at the 23-rd International Conference on Group Theory Methods in Physics, Dubna (Russia), August 2000 


\section{Introduction and main theorem}

As is well known, given two associative unital algebras $\mathcal{A}_{1}, \mathcal{A}_{2}$ (over the field $\mathbb{C}$, say), there is an obvious way to build a new algebra $\mathcal{A}$ which is as a vector space the tensor product $\mathcal{A}=\mathcal{A}_{1} \otimes_{\mathbb{C}} \mathcal{A}_{2}$ of the two vector spaces (over the same field) and has a product law such that $\mathcal{A}_{1} \otimes \mathbf{1}$ and $\mathbf{1} \otimes \mathcal{A}_{2}$ are subalgebras isomorphic to $\mathcal{A}_{1}$ and $\mathcal{A}_{2}$ respectively: one just completes the product law by postulating the trivial commutation relations

$$
\left(\mathbf{1} \otimes a_{2}\right)\left(a_{1} \otimes \mathbf{1}\right)=\left(a_{1} \otimes \mathbf{1}\right)\left(\mathbf{1} \otimes a_{2}\right)
$$

for any $a_{1} \in \mathcal{A}_{1}, a_{2} \in \mathcal{A}_{2}$. The resulting algebra is the ordinary tensor product algebra. With a standard abuse of notation we shall denote in the sequel $a_{1} \otimes a_{2}$ by $a_{1} a_{2}$ for any $a_{1} \in \mathcal{A}_{1}, a_{2} \in \mathcal{A}_{2}$; consequently (而) becomes

$$
a_{2} a_{1}=a_{1} a_{2}
$$

If $\mathcal{A}_{1}, \mathcal{A}_{2}$ are module algebras of a Lie algebra $\mathrm{g}$, and we require $\mathcal{A}$ to be too, then (2) has no alternative, because any $g \in \mathbf{g}$ acts as a derivation on the (algebra as well as tensor) product of any two elements, or, in Hopf algebra language, because the coproduct $\Delta(g)=g_{(1)} \otimes g_{(2)}$ (at the rhs we have used Sweedler notation) of the Hopf algebra $H \equiv U \mathbf{g}$ is cocommutative. In this paper we shall work with right-module algebras (instead of left ones), and denote by $\triangleleft:\left(a_{i}, g\right) \in \mathcal{A}_{i} \times H \rightarrow a_{i} \triangleleft g \in \mathcal{A}_{i}$ the right action; the reason is that they are equivalent to left comodule algebras, which are used in much of the literature. In Ref. [9] we give also the corresponding formulae for the left module algebras. We recall that a right action $\triangleleft:(a, g) \in \mathcal{A} \times H \rightarrow a \triangleleft g \in \mathcal{A}$ by definition fulfills

$$
\begin{aligned}
& a \triangleleft\left(g g^{\prime}\right)=(a \triangleleft g) \triangleleft g^{\prime}, \\
& \left(a a^{\prime}\right) \triangleleft g=\left(a \triangleleft g_{(1)}\right)\left(a^{\prime} \triangleleft g_{(2)}\right) .
\end{aligned}
$$

If we take as Hopf algebra $H$ a quasitriangular noncocommutative one like the quantum group $U_{q} \mathbf{g}$, as $\mathcal{A}_{i}$ some $H$-module algebras, and we require

$\mathcal{A}$ to be a $H$-module algebra too, then (2) has to be replaced by one of the formulae

$$
\begin{aligned}
& a_{2} a_{1}=\left(a_{1} \triangleleft \mathcal{R}^{(1)}\right)\left(a_{2} \triangleleft \mathcal{R}^{(2)}\right), \\
& a_{2} a_{1}=\left(a_{1} \triangleleft \mathcal{R}^{-1(2)}\right)\left(a_{2} \triangleleft \mathcal{R}^{-1(1)}\right) .
\end{aligned}
$$


This yields instead of $\mathcal{A}$ two different braided tensor product algebras [10, 11], which we shall call $\mathcal{A}^{+}=\mathcal{A}_{1} \underline{\otimes}^{+} \mathcal{A}_{2}$ and $\mathcal{A}^{-}=\mathcal{A}_{1} \otimes^{-} \mathcal{A}_{2}$ respectively. Here $\mathcal{R} \equiv \mathcal{R}^{(1)} \otimes \mathcal{R}^{(2)} \in H^{+} \otimes H^{-}$denotes the so-called universal $R$-matrix of $H \equiv[6], \mathcal{R}^{-1}$ its inverse, and $H^{ \pm}$denote the Hopf positive and negative Borel subalgebras of $H$. If in particular $H$ is triangular, then $\mathcal{R}^{-1}=\mathcal{R}_{21}$, $\mathcal{A}^{+}=\mathcal{A}^{-}$, and one has just one braided tensor product algebra. In any case, both $\mathcal{A}^{+}$and $\mathcal{A}^{-}$go to the ordinary tensor product algebra $\mathcal{A}$ in the limit $q \rightarrow 1$, because in this limit $\mathcal{R} \rightarrow \mathbf{1} \otimes \mathbf{1}$.

The braided tensor product is a particular example of a more general notion, that of a crossed (or twisted) tensor product [1] of two unital associative algebras.

In view of (5) or (6) studying representations of $\mathcal{A}^{ \pm}$is a more difficult task than just studying the representations of $\mathcal{A}_{1}, \mathcal{A}_{2}$ and taking their tensor products. The degrees of freedom of $\mathcal{A}_{1}, \mathcal{A}_{2}$ are so to say "coupled". One might ask whether one can "decouple" them by a transformation of generators. As shown in Ref. [9], the answer is positive if there respectively exists an algebra homomorphism $\varphi_{1}^{+}$or an algebra homomorphism $\varphi_{1}^{-}$

$$
\varphi_{1}^{ \pm}: \mathcal{A}_{1} \rtimes H^{ \pm} \rightarrow \mathcal{A}_{1}
$$

acting as the identity on $\mathcal{A}_{1}$, namely for any $a_{1} \in \mathcal{A}_{1}$

$$
\varphi_{1}^{ \pm}\left(a_{1}\right)=a_{1} .
$$

(Here $\mathcal{A}_{1} \rtimes H^{ \pm}$denotes the cross product between $\mathcal{A}_{1}$ and $H^{ \pm}$). In other words, this amounts to assuming that $\varphi_{1}^{+}\left(H^{+}\right)\left[\right.$resp. $\left.\varphi_{1}^{-}\left(H^{-}\right)\right]$provides a realization of $H^{+}$(resp. $H^{-}$) within $\mathcal{A}_{1}$. In this report we summarize the main results of Ref. [9]. The basic one is

Theorem 1 [9]. Let $\{H, \mathcal{R}\}$ be a quasitriangular Hopf algebra and $H^{+}, H^{-}$ be Hopf subalgebras of $H$ such that $\mathcal{R} \in H^{+} \otimes H^{-}$. Let $\mathcal{A}_{1}, \mathcal{A}_{2}$ be respectively a $\mathrm{H}^{+}$- and a $\mathrm{H}^{-}$-module algebra, so that we can define $\mathcal{A}^{+}$as in (5), and $\varphi_{1}^{+}$be a homomorphism of the type (17), (8), so that we can define the map $\chi^{+}: \mathcal{A}_{2} \rightarrow \mathcal{A}^{+}$by

$$
\chi^{+}\left(a_{2}\right):=\varphi_{1}^{+}\left(\mathcal{R}^{(1)}\right)\left(a_{2} \triangleleft \mathcal{R}^{(2)}\right) .
$$

Alternatively, let $\mathcal{A}_{1}, \mathcal{A}_{2}$ be respectively a $\mathrm{H}^{-}$- and a $\mathrm{H}^{+}$-module algebra, so that we can define $\mathcal{A}^{-}$as in (6), and $\varphi_{1}^{-}$be a homomorphism of the type (17), (8), so that we can define the map $\chi^{-}: \mathcal{A}_{2} \rightarrow \mathcal{A}^{-}$by

$$
\chi^{-}\left(a_{2}\right):=\varphi_{1}^{-}\left(\mathcal{R}^{-1(2)}\right)\left(a_{2} \triangleleft \mathcal{R}^{-1(1)}\right) .
$$


In either case $\chi^{ \pm}$are then injective algebra homomorphisms and

$$
\left[\chi^{ \pm}\left(a_{2}\right), \mathcal{A}_{1}\right]=0,
$$

namely the subalgebras $\tilde{\mathcal{A}}_{2}^{ \pm}:=\chi^{ \pm}\left(\mathcal{A}_{2}\right) \approx \mathcal{A}_{2}$ commute with $\mathcal{A}_{1}$. Moreover $\mathcal{A}^{ \pm}=\mathcal{A}_{1} \otimes \tilde{\mathcal{A}}_{2}^{ \pm}$.

The last equality means that $\mathcal{A}^{ \pm}$are respectively equal to the ordinary tensor product algebra of $\mathcal{A}_{1}$ with the subalgebras $\tilde{\mathcal{A}}_{2}^{ \pm} \subset \mathcal{A}^{ \pm}$, which are isomorphic to $\mathcal{A}_{2}$ ! $\chi^{+}, \chi^{-}$will be called "unbraiding" maps.

We recall the content of the hypotheses stated in the theorem. The algebra $\mathcal{A}_{1} \rtimes H^{ \pm}$as a vector space is the tensor product $\mathcal{A}_{1} \otimes_{\mathbb{C}} H^{ \pm}$, as an algebra it has subalgebras $\mathcal{A}_{1} \otimes \mathbf{1}, \mathbf{1} \otimes H$ and has cross commutation relations

$$
a_{1} g=g_{(1)}\left(a_{1} \triangleleft g_{(2)}\right),
$$

for any $a_{1} \in \mathcal{A}_{1}$ and $g \in H^{ \pm}$. $\varphi_{1}^{ \pm}$being an algebra homomorphism means that for any $\xi, \xi^{\prime} \in \mathcal{A}_{1} \triangleleft H^{ \pm} \varphi_{1}^{ \pm}\left(\xi \xi^{\prime}\right)=\varphi_{1}^{ \pm}(\xi) \varphi_{1}^{ \pm}\left(\xi^{\prime}\right)$. Applying $\varphi_{1}^{ \pm}$to both sides of (12) we find $a \varphi^{ \pm}(g)=\varphi^{ \pm}\left(g_{(1)}\right)\left(a \triangleleft g_{(2)}\right)$.

Of course, we can use the above theorem iteratively to completely unbraid the braided tensor product algebra of an arbitrary number $M$ of copies of $\mathcal{A}_{1}$. We end up with

Corollary 1 If $\mathcal{A}_{1}$ is a (right-) module algebra of the Hopf algebra $H$ and there exists an algebra homomorphism $\varphi_{1}^{+}$of the type (1), (8), then there is an algebra isomorphism.

$$
\underbrace{\mathcal{A}_{1} \underline{\otimes}^{+} \ldots \underline{\otimes}^{+} \mathcal{A}_{1}}_{M \text { times }} \approx \underbrace{\mathcal{A}_{1} \otimes \ldots \otimes \mathcal{A}_{1}}_{M \text { times }} .
$$

An analogous claim holds for the second braided tensor product if there exists a $\operatorname{map} \varphi_{1}^{-}$.

\section{The unbraiding under the $*$-structures}

$\mathcal{A}^{+}$(as well as $\mathcal{A}^{-}$) is a $*$-algebra if $H$ is a Hopf $*$-algebra, $\mathcal{A}_{1}, \mathcal{A}_{2}$ are $H$ module $*$-algebras (we shall use the same symbol $*$ for the $*$-structure on all algebras $H, \mathcal{A}_{1}$, etc.), and

$$
\mathcal{R}^{*}=\mathcal{R}^{-1}
$$


(here $\mathcal{R}^{*}$ means $\mathcal{R}^{(1) *} \otimes \mathcal{R}^{(2) *}$ ). In the quantum group case (14) requires $|q|=1$. Under the same assumptions also $\mathcal{A}_{1} \triangleleft H$ is a $*$-algebra. If $\varphi_{1}^{ \pm}$exist setting $\varphi_{1}^{\prime \pm}:=* \circ \varphi_{1}^{ \pm} \circ *$ we realize that also $\varphi_{1}^{\prime \pm}$ are algebra homomorphisms of the type (7), (8). If such homomorphisms are uniquely determined, we conclude that $\varphi_{1}^{ \pm}$are $*$-homomorphisms. More generally, one may be able to choose $\varphi_{1}^{ \pm}$as $*$-homomorphisms. How do the corresponding $\chi^{ \pm}$behave under $*$ ?

Proposition 1 [9]. Assume that the conditions of Theorem [1 for defining $\chi^{+}$(resp. $\chi^{-}$) are fulfilled. If $\mathcal{R}^{*}=\mathcal{R}^{-1}$ and $\varphi_{1}^{+}$(resp. $\varphi_{1}^{-}$) is a *-homomorphism then $\chi^{+}$(resp. $\left.\chi^{-}\right)$is, too. Consequently, $\mathcal{A}_{1}, \tilde{\mathcal{A}}_{2}^{ \pm}$are closed under $*$.

\section{Applications}

In this section we illustrate the application of Theorem 1 and Corollary 1 to some algebras $H, \mathcal{A}_{i}$ for which homomorphisms $\varphi_{1}^{ \pm}$are known. $H$ will be the quantum group $U_{q} s l(N)$ or $U_{q} \mathrm{so}(N)$, and $\mathcal{A}_{1}$ is the $U_{q} s l(N)$ - or $U_{q} \mathrm{so}(N)$ covariant Heisenberg algebra (Section 3.1.), the $U_{q} \mathrm{so}(N)$-covariant quantum space/sphere (Section 3.2.). In Ref. [9] we have treated also the $U_{q} s o(3)$ covariant $q$-fuzzy sphere. As generators of $\mathrm{H}$ it will be convenient in either case to use the Faddeev-Reshetikhin-Takhtadjan (FRT) generators [7] $\mathcal{L}^{+a} \in$ $H^{+}$and $\mathcal{L}_{l}^{-a} \in H^{-}$. They are related to $\mathcal{R}$ by

$$
\mathcal{L}^{+a}:=\mathcal{R}^{(1)} \rho_{l}^{a}\left(\mathcal{R}^{(2)}\right) \quad \mathcal{L}_{l}^{-a}:=\rho_{l}^{a}\left(\mathcal{R}^{-1(1)}\right) \mathcal{R}^{-1(2)},
$$

where $\rho_{l}^{a}(g)$ denote the matrix elements of $g \in U_{q} \mathbf{g}$ in the fundamental $N$ dimensional representation $\rho$ of $U_{q} \mathbf{g}$. In fact they provide, together with the square roots of the elements $\mathcal{L}_{i}^{ \pm i}$, a (overcomplete) set of generators of $U_{q} \mathbf{g}$.

\subsection{Unbraiding 'chains' of braided Heisenberg alge- bras}

In this subsection we consider the braided tensor product of $M \geq 2$ copies of the $U_{q} \mathbf{g}$-covariant deformed Heisenberg algebras $\mathcal{D}_{\epsilon, \mathbf{g}}, \mathbf{g}=\operatorname{sl}(N), s o(N)$. Such algebras have been introduced in Ref. [13, 14, 2]. They are unital associative algebras generated by $x^{i}, \partial_{j}$ fulfilling the relations

$$
\mathcal{P}_{a h k}{ }^{i j} x^{h} x^{k}=0, \quad \mathcal{P}_{a h k}{ }^{i j} \partial_{j} \partial_{i}=0, \quad \partial_{i} x^{j}=\delta_{j}^{i}+(q \gamma \hat{R})_{i h}{ }_{i h} x^{h} \partial_{k},
$$


where $\gamma=q^{\frac{1}{N}}, 1$ respectively for $\mathbf{g}=\operatorname{sl}(N), s o(N)$, and the exponent $\epsilon$ can take either value $\epsilon=1,-1$. $\hat{R}$ denotes the braid matrix of $U_{q} \mathbf{g}$ [given in formulae (27)], and the matrix $\mathcal{P}_{a}$ is the deformed antisymmetric projector appearing in the decomposition (28) of the latter. The coordinates $x^{i}$ transform according to the fundamental $N$-dimensional representation $\rho$ of $U_{q} \mathbf{g}$, whereas the 'partial derivatives' transform according the contragredient representation,

$$
x^{i} \triangleleft g=\rho_{j}^{i}(g) x^{j}, \quad \partial_{i} \triangleleft g=\partial_{h} \rho_{i}^{h}\left(S^{-1} g\right) .
$$

In our conventions the indices will take the values $i=1, \ldots, N$ if $\mathbf{g}=\operatorname{sl}(N)$, whereas if $\mathbf{g}=s o(N)$ they will take the values $i=-n, \ldots,-1,0,1, \ldots n$ for $N$ odd, and $i=-n, \ldots,-1,1, \ldots n$ for $N$ even; here $n:=\left[\frac{N}{2}\right]$ denotes the rank of $s o(N)$. We shall enumerate the different copies of $\mathcal{D}_{\epsilon, \mathbf{g}}$ by attaching to them an additional Greek index, e.g. $\alpha=1,2, \ldots, M$. The prescription (6) gives the following "cross" commutation relations between their respective generators $(\alpha<\beta)$.

$$
\begin{aligned}
x^{\alpha, i} x^{\beta, j} & =\hat{R}_{h k}^{i j} x^{\beta, h} x^{\alpha, k}, & \partial_{\alpha, i} \partial_{\beta, j} & =\hat{R}_{j i}^{k h} \partial_{\beta, h} \partial_{\alpha, k}, \\
\partial_{\alpha, i} x^{\beta, j} & =\hat{R}^{-1 j h} x^{\beta, k} \partial_{\alpha, h}, & \partial_{\beta, i} x^{\alpha, j} & =\hat{R}_{i k}^{j h} x^{\alpha, k} \partial_{\beta, h} .
\end{aligned}
$$

Algebra homomorphisms $\varphi_{1}: \mathcal{A}_{1} \rtimes H \rightarrow \mathcal{A}_{1}$, for $H=U_{q} \mathbf{g}$ and $\mathcal{A}_{1}$ equal to (a suitable completion of) $\mathcal{D}_{\epsilon, \mathbf{g}}$ have been constructed in Ref. [8, 5]. This is the $q$-analog of the well-known fact that the elements of $\mathbf{g}$ can be realized as "vector fields" (first order differential operators) on the corresponding $\mathbf{g}$ covariant (undeformed) space, e.g. $\varphi_{1}\left(E_{j}^{i}\right)=x^{i} \partial_{j}-\frac{1}{N} \delta_{j}^{i}$ in the $\mathbf{g}=s l(N)$ case. The maps $\varphi_{1}^{ \pm}$needed to apply Theorem 11 are simply the restrictions to $\mathcal{A}_{1} \triangleleft H^{ \pm}$of $\varphi_{1}$ of Ref. [8, 5].

The unbraiding procedure is recursive. We just describe the first step, which consists of using the homomorphism $\varphi_{1}^{ \pm}$to unbraid the first copy from the others. According to the main theorem, if we set

$$
\begin{aligned}
y^{1, i} & \equiv x^{1, i} \quad \partial_{y, 1, a} \equiv \partial_{1, a} \\
y^{\alpha, i} & \equiv \chi^{-}\left(x^{\alpha, i}\right)=\varphi_{1}\left(\mathcal{R}^{-1(2)}\right) \rho_{j}^{i}\left(\mathcal{R}^{-1(1)}\right) x^{\alpha, j}=\varphi_{1}\left(\mathcal{L}_{j}^{-i}\right) x^{\alpha, j} \\
\partial_{y, \alpha, a} & \equiv \chi^{-}\left(\partial_{\alpha, a}\right)=\varphi_{1}\left(S \mathcal{R}^{-1(2)}\right) \rho_{a}^{d}\left(\mathcal{R}^{-1(1)}\right) \partial_{\alpha, d}=\varphi_{1}\left(S \mathcal{L}^{-d}\right) \partial_{\alpha, d}
\end{aligned}
$$

with $\alpha>1$. By Theorem $11 y^{1, i} \equiv x^{1, i}$ and $\partial_{y, 1, i} \equiv \partial_{1, i}$ will commute with $y^{2, i}, \ldots, y^{M, i}$ and $\partial_{y, 2, i}, \ldots, \partial_{y, M, i}$. As we see, the FRT generators are special because they appear in the redefinitions (20-21). The explicit expression of 
$\varphi_{1}\left(\mathcal{L}_{j}^{-i}\right)$ in terms of $x^{1, i}, \partial_{1, a}$ for $U_{q} s l(2), U_{q} s o(3)$ has been given in Ref. [9]. For different values of $N$ it can be found from the results of Ref. [8, 5] by passing from the generators adopted there to the FRT generators.

By completely analogous arguments one determines the alternative unbraiding procedure for the braided tensor product stemming from prescription (5).

$\mathcal{A}_{1} \triangleleft H$ is a $*$-algebra and the map $\varphi_{1}$ is a $*$-homomorphism both for $q$ real and $|q|=1$. But $\varphi_{1}^{ \pm}$are $*$-homomorphisms only for $|q|=1$. In the latter case the $*$-structure of $\mathcal{A}_{1}$ is

$$
\left(x^{i}\right)^{*}=x^{i}, \quad\left(\partial_{i}\right)^{*}=-q^{ \pm N} g^{k h} g_{k i} \partial_{h}
$$

Applying Proposition 1 in the latter case we find that $*$ maps $\mathcal{A}_{1}$ as well as each of the commuting subalgebras $\tilde{\mathcal{A}}_{i}^{ \pm}$into itself.

\subsection{Unbraiding 'chains' of braided quantum Euclidean spaces or spheres}

In this section we consider the braided tensor product of $M \geq 2$ copies of the quantum Euclidean space $\mathbb{R}_{q}^{N}$ [7] (the $U_{q} \mathrm{so}(N)$-covariant quantum space), i.e. of the unital associative algebra generated by $x^{i}$ fulfilling the relations $(16)_{1}$, or of the quotient space of $\mathbb{R}_{q}^{N}$ obtained by setting $r^{2}:=$ $x^{i} x_{i}=1$ [the quantum $(N-1)$-dimensional sphere $S_{q}^{N-1}$ ]. (Thus, these will be subalgebras of the Heisenberg algebras $\mathcal{D}_{+, s o(N)}, \mathcal{D}_{+, s o(N)}$ considered in the previous subsection). Again, the multiplet $\left(x^{i}\right)$ carries the fundamental $N$ dimensional representation $\rho$ of $U_{q} \mathrm{so}(N)$. As before, we shall enumerate the different copies of the quantum Euclidean space or sphere by attaching an additional Greek index to them, e.g. $\alpha=1,2, \ldots, M$. The prescription (6) gives the cross commutation relations $(18)_{1}$.

According to Ref. [3], to define $\varphi_{1}^{ \pm}($for $q \neq 1)$ one actually needs a slightly enlarged version of $\mathbb{R}_{q}^{N}$ (or $S_{q}^{N-1}$ ). One has to introduce some new generators $\sqrt{r_{a}}$, with $0 \leq a \leq \frac{N}{2}$, together with their inverses $\left(\sqrt{r_{a}}\right)^{-1}$, requiring that

$$
r_{a}^{2}=\sum_{h=-a}^{a} x^{h} x_{h}=\sum_{h=-a}^{a} g_{h k} x^{h} x^{k}
$$

(note that, having set $n:=\left[\frac{N}{2}\right], r_{n}^{2}$ coincides with $r^{2}$, whereas for odd $N$ $r_{0}^{2}=\left(x^{0}\right)^{2}$, so we are adding also $\left(x^{0}\right)^{-1}$ as a new generator). In fact, the commutation relations involving these new generators can be fixed consistently, 
and turn out to be simply $q$-commutation relations. $r$ plays the role of 'deformed Euclidean distance' of the generic 'point' $\left(x^{i}\right)$ of $\mathbb{R}_{q}^{N}$ from the 'origin'; $r_{a}$ is the 'projection' of $r$ on the 'subspace' $x^{i}=0,|i|>a$. In the previous equation $g_{h k}$ denotes the 'metric matrix' of $S O_{q}(N), g_{i j}=g^{i j}=q^{-\rho_{i}} \delta_{i,-j}$, which is a $S O_{q}(N)$-isotropic tensor and a deformation of the ordinary Euclidean metric. Here, $\left(\rho_{i}\right):=\left(n-\frac{1}{2}, \ldots, \frac{1}{2}, 0,-\frac{1}{2}, \ldots, \frac{1}{2}-n\right)$ for $N$ odd, $\left(\rho_{i}\right):=(n-1, \ldots, 0,0, \ldots, 1-n)$ for $N$ even. $g_{i j}$ is related to the trace projector appearing in (28) by $\mathcal{P}_{t k l}^{i j}=\left(g^{s m} g_{s m}\right)^{-1} g^{i j} g_{k l}$. The extension of the action of $H$ to these extra generators is uniquely determined by the constraints the latter fulfil. In the case of even $N$ one needs to include also the FRT generators $\mathcal{L}^{+1}, \mathcal{L}^{-1}$ (which are generators of $H$ ) among the generators of $\mathcal{A}_{1}$. In appendix 3.2. we recall the explicit form of $\varphi_{1}^{ \pm}$in the present case. Note that the maps $\varphi_{1}^{ \pm}$have no analog in the "undeformed" case $(q=1)$, because $\mathcal{A}_{1}$ is abelian, whereas $H$ is not.

The unbraiding procedure is recursive. The first step consists of using the homomorphism $\varphi_{1}^{ \pm}$found in Ref. [3] to unbraid the first copy from the others. Following Theorem 1, we perform the change of generators $(19)_{1}$, (20) in $\mathcal{A}^{-}$. In view of formula (29) we thus find

$$
y^{1, i}:=x^{1, i}, \quad y^{\alpha, i}:=g^{i h}\left[\mu_{h}^{1}, x^{1, k}\right]_{q} g_{k j} x^{\alpha, j}, \quad \alpha>1 .
$$

The suffix 1 in $\mu_{a}^{1}$ means that the special elements $\mu_{a}$ defined in (30) must be taken as elements of the first copy. In view of (30) we see that $g^{i h}\left[\mu_{h}^{1}, x^{1, k}\right]_{q} g_{k j}$ are rather simple polynomials in $x^{i}$ and $r_{a}^{-1}$, homogeneous of total degree 1 in the coordinates $x^{i}$ and $r_{a}$. Using the results given in the appendix we give now the explicit expression of (24) 2 for $N=3$ :

$$
\begin{aligned}
y^{\alpha,-} & =-q h \gamma_{1} \frac{r}{x^{0}} x^{\alpha,-} \\
y^{\alpha, 0} & =\sqrt{q}(q+1) \frac{1}{x^{0}} x^{+} x^{\alpha,-}+x^{\alpha, 0} \\
y^{\alpha,+} & =\frac{\sqrt{q}(q+1)}{h \gamma_{1} r x^{0}}\left(x^{+}\right)^{2} x^{\alpha,-}+\frac{q^{-1}+1}{h \gamma_{1} r} x^{+} x^{\alpha, 0}-\frac{1}{q h \gamma_{1} r} x^{0} x^{\alpha,+}
\end{aligned}
$$

for any $\alpha=2, \ldots, M$. Here we have set $x^{i} \equiv x^{1, i}, h \equiv \sqrt{q}-1 / \sqrt{q}$, replaced for simplicity the values $-1,0,1$ of the indices by the ones $-, 0,+$ and denoted by $\gamma_{1} \in \mathbb{C}$ a free parameter. By Theorem $11 y^{1, i} \equiv x^{1, i}$ commutes with $y^{2, i}, \ldots, y^{M, i}$. 
The alternative unbraiding procedure for the braided tensor product algebra stemming from prescription (5) arises by iterating the change of generators

$$
y^{\prime M, i}:=x^{M, i} \quad y^{\prime \alpha, i}:=\varphi_{M}\left(\mathcal{L}_{j}^{+i}\right) x^{\alpha, j}=g^{i h}\left[\bar{\mu}_{h}^{M}, x^{M, k}\right]_{q^{-1}} g_{k j} x^{\alpha, j},
$$

$\alpha<M$. The special elements $\bar{\mu}_{a}$ are defined in (30), and suffix ${ }^{M}$ means that we must take $\bar{\mu}_{a}$ as an element of the $M$-th copy of $\mathbb{R}_{q}^{N}$ (or $S_{q}^{N-1}$ ). $y^{M, i} \equiv x^{M, i}$ commutes with $y^{1, i}, \ldots, y^{M-1, i}$.

When $|q|=1$, by a suitable choice (32) of $\gamma_{1}, \bar{\gamma}_{1}$, as well as of the other free parameters $\gamma_{a}, \bar{\gamma}_{a}$ appearing in the definitions of $\varphi^{ \pm}$for $N>3$, one can make $\varphi^{ \pm}$into $*$-homomorphisms. Applying Proposition 1 in the latter case we find that $*$ maps $\mathcal{A}_{1}$ as well as each of the commuting subalgebras $\tilde{\mathcal{A}}_{i}^{ \pm}$ into itself.

The braid matrix $\hat{R}$ is related to $\mathcal{R}$ by $\hat{R}_{h k}^{i j} \equiv R_{h k}^{j i}:=\left(\rho_{h}^{j} \otimes \rho_{k}^{i}\right) \mathcal{R}$. With the indices' convention described in sections 3.2., 3.1. $\hat{R}$ is given by

$$
\begin{gathered}
\hat{R}=q^{-\frac{1}{N}}\left[q \sum_{i} e_{i}^{i} \otimes e_{i}^{i}+\sum_{i \neq j} e_{i}^{j} \otimes e_{j}^{i}+k \sum_{i<j} e_{i}^{i} \otimes e_{j}^{j}\right] \\
\hat{R}=q \sum_{i \neq 0} e_{i}^{i} \otimes e_{i}^{i}+\sum_{\substack{i \neq j,-j \\
\text { or } i=j=0}} e_{i}^{j} \otimes e_{j}^{i}+q^{-1} \sum_{i \neq 0} e_{i}^{-i} \otimes e_{-i}^{i} \\
+k \sum_{i<j}\left(e_{i}^{i} \otimes e_{j}^{j}-q^{-\rho_{i}+\rho_{j}} e_{i}^{-j} \otimes e_{-i}^{j}\right)
\end{gathered}
$$

for $\mathbf{g}=\operatorname{sl}(N), s o(N)$ respectively. Here $e_{j}^{i}$ is the $N \times N$ matrix with all elements equal to zero except for a 1 in the $i$ th column and $j$ th row. The braid matrix of $s o(N)$ admits the orthogonal projector decomposition

$$
\hat{R}=q \mathcal{P}_{s}-q^{-1} \mathcal{P}_{a}+q^{1-N} \mathcal{P}_{t}
$$

$\mathcal{P}_{a}, \mathcal{P}_{t}, \mathcal{P}_{s}$ are the $q$-deformed antisymmetric, trace, trace-free symmetric projectors. There are just two projectors $\mathcal{P}_{a}, \mathcal{P}_{s}$ in decomposition of the braid matrix of $\operatorname{sl}(N)$. the latter is obtained from (28) just by deleting the third term.

We now recall the explicit form of maps $\varphi^{ \pm}$for the quantum Euclidean spaces or spheres, found in Ref. [3]. These are algebra homomorphisms $\varphi^{ \pm}: \mathbb{R}_{q}^{N} \rtimes U_{q}^{ \pm} s o(N) \rightarrow \mathbb{R}_{q}^{N}$. We introduce the short-hand notation $[A, B]_{x}=$ 
$A B-x B A$. The images of $\varphi^{-}$(resp. $\left.\varphi^{+}\right)$on the negative (resp. positive) FRT generators read

$$
\varphi^{-}\left(\mathcal{L}_{j}^{-i}\right)=g^{i h}\left[\mu_{h}, x^{k}\right]_{q} g_{k j}, \quad \quad \varphi^{+}\left(\mathcal{L}_{j}^{+i}\right)=g^{i h}\left[\bar{\mu}_{h}, x^{k}\right]_{q^{-1}} g_{k j},
$$

where

$$
\begin{array}{lll}
\mu_{0}=\gamma_{0}\left(x^{0}\right)^{-1} & \bar{\mu}_{0}=\bar{\gamma}_{0}\left(x^{0}\right)^{-1} & \text { for } N \text { odd } \\
\mu_{ \pm 1}=\gamma_{ \pm 1}\left(x^{ \pm 1}\right)^{-1} \mathcal{L}_{1}^{\mp 1} & \bar{\mu}_{ \pm 1}=\bar{\gamma}_{ \pm 1}\left(x^{ \pm 1}\right)^{-1} \mathcal{L}_{1}^{ \pm 1} & \text { for } N \text { even } \\
\mu_{a}=\gamma_{a} r_{|a|}^{-1} r_{|a|-1}^{-1} x^{-a} & \bar{\mu}_{a}=\bar{\gamma}_{a} r_{|a|}^{-1} r_{|a|-1}^{-1} x^{-a} & \text { otherwise }
\end{array}
$$

and $\gamma_{a}, \bar{\gamma}_{a} \in \mathbb{C}$ are normalization constants fulfilling the conditions

$$
\begin{aligned}
& \gamma_{0}=-q^{-\frac{1}{2}} h^{-1} \quad \bar{\gamma}_{0}=q^{\frac{1}{2}} h^{-1} \quad \text { for } N \text { odd, }
\end{aligned}
$$

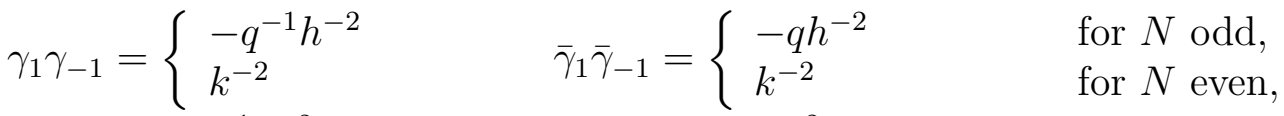

$$
\begin{aligned}
& \gamma_{a} \gamma_{-a}=-q^{-1} k^{-2} \omega_{a} \omega_{a-1} \quad \bar{\gamma}_{a} \bar{\gamma}_{-a}=-q k^{-2} \omega_{a} \omega_{a-1} \quad \text { for } a>1 .
\end{aligned}
$$

Here $k:=q-1 / q, \omega_{a}:=\left(q^{\rho_{a}}+q^{-\rho_{a}}\right)$. Incidentally, for odd $N$ one can choose the free parameters $\gamma_{a}, \bar{\gamma}_{a}$ in such a way that $\varphi^{+}, \varphi^{-}$can be 'glued' into an algebra homomorphism $\varphi: \mathbb{R}_{q}^{N} \rtimes U_{q} \operatorname{so}(N) \rightarrow \mathbb{R}_{q}^{N}$ [3]. When $|q|=1$, the $*$-structure is given by $\left(x^{i}\right)^{*}=x^{i}[$ see $(22)]$. It turns out that $\varphi^{ \pm}$are $*$-homomorphisms if, in addition,

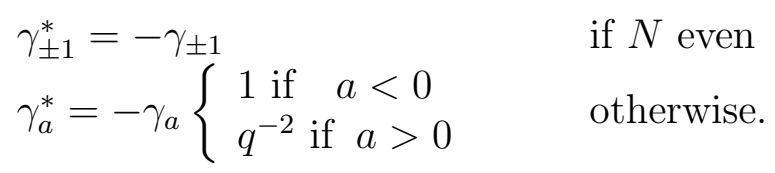

\section{References}

[1] A. Van Daele and S. Van Keer, Compositio Mathematica 91, 201 (1994). A. Borowiec, W. Marcinek, "On crossed product of algebras", mathph/0007031, and references therein; "Hopf modules and their duals", math.QA/0007151.

[2] U. Carow-Watamura, M. Schlieker, S. Watamura, Z. Physik C 49 (1991) 439. 
[3] B. L. Cerchiai, G. Fiore, J. Madore, " Geometrical Tools for Quantum Euclidean Spaces", to appear in Commun. Math. Phys., math.QA/0002007

[4] B.L. Cerchiai, J. Madore, S. Schraml, J. Wess, Eur.Phys.J. C16 (2000), 169-180.

[5] C.-S. Chu, B. Zumino, "Realization of vector fields fro quantum groups as pseudodifferential operators on quantum spaces", Proc. XX Int. Conf. on Group Theory Methods in Physics, Toyonaka (Japan), 1995, and qalg/9502005.

[6] V. Drinfeld, "Quantum groups," in I.C.M. Proceedings, Berkeley, (1986) p. 798 .

[7] L.D. Faddeev, N.Y. Reshetikhin, L. Takhtadjan, Leningrad Math. J. 1 (1990), 193.

[8] G. Fiore, Commun. Math. Phys. 169 (1995), 475-500.

[9] G. Fiore, H. Steinacker, J. Wess "Unbraiding the braided tensor product", Preprint 00-30 Dip. Matematica e Applicazioni, Università di Napoli, math/0007174.

[10] A. Joyal, R. Streat, Braided Monoidal Categories, Mathematics Reports 86008, Macquarie University, 1986.

[11] S. Majid, Int. J. Mod. Phys. A5, 1 (1990); J. Algebra 130, 17 (1990); Lett. Math. Phys. 22, 167 (1991); J. Algebra 163, 191 (1994). For a review: S. Majid, Foundations of Quantum Groups, Cambridge Univ. Press (1995); and references therein.

[12] O. Ogievetsky, Lett. Math. Phys. 24 (1992), 245.

[13] W. Pusz, S. L. Woronowicz, Rep. Math. Phys. 27 (1989), 231.

[14] J. Wess, B. Zumino, Nucl. Phys. (Proc. Suppl.) 18B (1990) 302. 